\title{
The Relationship between Coherent Integration and Doppler Spectral Processing of Weather Radar Echoes
}

\author{
PAUL L. SMITH* \\ Department of Meteorology, University of Helsinki, SF-00100 Helsinki, Finland
}

25 June 1986 and 26 January 1987

\section{Introduction}

Signal processing is an important aspect of modern radar systems. Doppler processing is often used to extract velocity information from weather echoes, while wind profilers also employ a form of integration which approximates coherent integration (see Gossard and Strauch, 1983, or Strauch et al., 1984). Both this "quasicoherent integration" and Doppler spectrum analysis are forms of coherent signal processing, and their relationship to coherent integration is of interest. The purpose of this note is to discuss that relationship; related material can be found in Chapter 11 of Gossard and Strauch (1983).

To carry out coherent integration of the output of a receiver requires summing output values in such a way that the signal ${ }^{1}$ components combine in phase while the noise components (with random phases) do not. For a signal of constant amplitude, coherent integration would increase the signal-to-noise ratio in direct proportion to the number of output values summed. However, coherent integration to aid in separating a signal from noise is possible only when the phase of the signal, as distinct from that of the output (signal + noise), is known. Because echoes from meteorological targets have random phases, this condition cannot be realized in weather radar systems. Consequently, true coherent integration cannot be carried out in such systems.

\section{Quasi-coherent integration}

However, as shown by the wind profiler work (Strauch et al., 1984), a quasi-coherent integration of the receiver output can be performed when the signal phase changes very slowly over the integration time. This is possible with slow-moving targets at long radar wavelengths. The need to know the signal phase is circumvented by summing the sampled complex video

\footnotetext{
* Present affiliation: Institute of Atmospheric Sciences, South Dakota School of Mines and Technology, Rapid City, SD 57701.

${ }^{1}$ Here "signal" means "echo;" the receiver output comprises signal plus noise.
}

outputs of an I-Q receiver separately, causing the signal components to combine roughly in phase if the variation of the signal phase over the integration time is not too great.

As noted in Strauch et al. (1984) or Smith (1986), applicability of this quasi-coherent integration is limited to the shorter of two time intervals:

(i) The interval over which the phase variation of the signal, due to the mean radial motion of the scatterers, is not too great. This interval is just $0.1855 \lambda /\left|\bar{v}_{r}\right|$, where $\lambda$ is the radar wavelength and $\bar{v}_{r}$ is the mean Doppler velocity (see Appendix).

(ii) The signal decorrelation time $t_{i}$, which is approximately $\lambda /\left(4 \sqrt{\pi} \sigma_{v}\right) \approx 0.14 \lambda / \sigma_{v}$. Here $\sigma_{v}$ is the standard deviation of the Doppler velocity spectrum (Nathanson, 1969; Walker et al., 1980).

The numerical factor is about the same in either case, so the limitation will be established by the larger of $\left|\bar{v}_{r}\right|$ or $\sigma_{v}$. In most cases, $v_{r}$ would be the governing quantity, but there could be exceptions.

Consider these time limitations, for typical mean Doppler velocities and spectrum widths, in comparison to typical weather radar pulse repetition periods. For example, at $10-\mathrm{cm}$ wavelength with a mean Doppler velocity of $20 \mathrm{~m} \mathrm{~s}^{-1}$, the available time for quasi-coherent integration would be less than $1 \mathrm{~ms}$. Such values show that quasi-coherent integration is likely to be applicable only for wavelengths longer than those ordinarily used in weather radars. If a rough estimate of the mean radial velocity were available, it would be possible to extend the quasi-coherent integration time by inserting a compensating, progressive pulse-to-pulse phase shift before summing the receiver output samples. As noted in section 3, that is essentially what occurs during Doppler spectral processing.

The principal benefit of quasi-coherent integration would be to enhance the integrated signal-to-noise ratio. That would enhance the system sensitivity, as discussed in Smith (1986). As indicated by the analysis in the Appendix, the increase is less than would be obtainable with true coherent integration. However, with favorable combinations of system parameters, the 
difference will be small. Wind profilers also make use of quasi-coherent integration to reduce the amount of computation required in subsequent processing of the receiver output (Gossard and Strauch, 1983).

As noted by Gossard and Strauch (1983), broadband modulation of the transmitted signal can be employed in a variety of interesting "pulse compression" radar techniques (e.g., FM-CW radar). Those techniques involve coherent integration of the echoes within the pulse repetition (modulation would be a better term) period. They are of interest in radar meteorology because of their potential for greater sensitivity, improved range resolution, or enhancement of sample size for reflectivity estimates. However, the applicability of such techniques would also be restricted to the shorter of the two time intervals described above.

\section{Doppler spectral processing}

Doppler processing of the receiver output involves much weaker constraints on the signal phase characteristics. The phase does not have to be known, and unambiguous Doppler velocities can often be determined even if the signal phase changes by nearly as much as $180^{\circ}$ between successive pulses. Furthermore, Doppler processing is commonly applied to samples of the receiver output acquired over a time long compared to the signal decorrelation time. Consequently, the limitations on coherent integration discussed in the previous section would seem not to be applicable to Doppler processing.

Nevertheless, Doppler spectrum analysis is still a form of coherent signal processing. An informative relationship can be developed by considering the improvement in signal-to-noise ratio associated with spectral processing. According to Gossard and Strauch (1983), that improvement is a factor

$$
F_{I}=\frac{T_{d}}{T_{r}} \operatorname{erf}\left(\frac{\lambda}{4 \sqrt{2} T_{d} \sigma_{v}}\right)
$$

where

$T_{d}$ dwell time used to collect data for a spectrum analysis,

$T_{r}$ pulse repetition period,

$\sigma_{v}$ standard deviation of the Doppler velocity spectrum, assumed Gaussian,

and $\operatorname{erf}(x)$ represents the error function. This improvement approaches a limiting value, in the usual situations with dwell times long enough to permit resolving the Doppler spectrum, of

$$
F_{I} \approx \frac{\lambda}{2 \sqrt{2 \pi} T_{r} \sigma_{v}}
$$

As noted earlier, the signal decorrelation time $t_{i}$ is approximately $\lambda /\left(4 \sqrt{\pi} \sigma_{v}\right)$. Consequently, (2) can be written as

$$
F_{I} \approx \sqrt{2} t_{i} / T_{r}
$$

But $t_{i} / T_{r}$ is just the number of output samples (i.e., pulses) obtained during the signal decorrelation time. Therefore, (3) indicates that the improvement in signalto-noise ratio accompanying spectral processing is roughly equivalent to that obtainable from coherent integration over the signal decorrelation time. Thus, it can be regarded as an indication of the amount of coherent integration involved in the Doppler spectrum processing.

This is not a "something-for-nothing" operation, because to resolve the spectrum (say, $\Delta v=\lambda / 2 T_{d} \leqslant \sigma_{v}$ ) requires a dwell time of at least

$$
T_{d}(\min )=\frac{\lambda}{2 \sigma_{v}} \approx 3.5 t_{i} .
$$

The total number of pulses involved is therefore at least $3.5 t_{i} / T_{r}$, so spectral processing also has less effect on the signal-to-noise ratio than true coherent integration. The fact that data acquisition for the Doppler processing may extend over an even longer dwell time provides no further enhancement of the signal-to-noise ratio (as observed by Gossard and Strauch, 1983). The additional dwell time merely provides velocity resolution finer than $\sigma_{v}$, as well as improved reflectivity estimates.

Professor Timo Puhakka (personal communication) has suggested the explanation for this property of spectral processing. Let the complex receiver output samples corresponding to a particular range be $E_{r}\left(m T_{r}\right)$, where $m$ has integer values $0 \leqslant m \leqslant(M-1), M$ being the total number of samples. The discrete Fourier transform of this sequence of samples at intervals of $T_{r}$ is obtained as

$$
F(k \Delta v)=\sum_{m=0}^{M-1} E_{r}\left(m T_{r}\right) e^{-j 2 \pi k m / M}
$$

where $\Delta v=\lambda / 2 T_{d}$ and $k$ has integer values $(1-M / 2)$ $\leqslant k \leqslant M / 2$. For any given velocity bin $k$, this calculation can be interpreted as the multiplication of the successive time samples $E_{r}\left(m T_{r}\right)$ by a progressive phase shift of $(2 \pi k)(m / M)$. That bin for which $(k \Delta v)$ most closely approximates the mean Doppler velocity of the target, i.e., for which $k \Delta v \approx \bar{v}_{r}$, will involve an incremental phase shift of just $2 \pi \bar{v}_{r} / M \Delta v=\pi \bar{v}_{r} / v_{\max }$ $=4 \pi\left(\bar{v}_{r} T_{r}\right) / \lambda$ per pulse, where $v_{\max }=\lambda / 4 T_{r}$ is the maximum unambiguous Doppler velocity. That will exactly compensate for the increment of echo phase due to motion of the target between pulses. Consequently the phase-shifted samples in the summation (5) will combine the echoes in phase, i.e., coherently, for that velocity bin.

The $M$ velocity bins apply all possible increments of phase shift per pulse (modulo $2 \pi$ ) and assure that one of the bins (if the Doppler spectrum is not too wide) will correspond to the correct value. The process will continue until the relative motion of the scatterers (as reflected by the spectrum width) destroys the co- 
herence of the echo. That introduces a random phase shift into the echo, and occurs after a time of the order of $t_{i}$.

Spectral processing will usually provide more signalto-noise improvement than would be obtainable by carrying out only quasi-coherent integration as discussed in section 2 . The reason is that, as noted there, the time limitation for such quasi-coherent integration is more likely to be established by mean-velocity considerations than by the signal decorrelation time. For spectral processing, on the other hand, the decorrelation time governs the amount of coherent integration. Under suitable conditions (which are designed into the wind profilers), the two types of signal processing can be carried out in sequence to obtain the full signal-tonoise improvement with less computational effort.

Acknowledgments. This work was carried out while the author was serving as a Fulbright Lecturer in Radar Meteorology at the University of Helsinki. Thanks are due to the U.S. Information Agency, the Finland-U.S. Educational Exchange Commission, the University of Helsinki Department of Meteorology, and the South Dakota School of Mines and Technology for their support. Reviewers of the manuscript provided helpful suggestions.

\section{APPENDIX}

\section{Phase Limit for Quasi-coherent Integration}

Suppose complex (I-Q) receiver output samples from a given range are summed as the phase angle $\phi$ of a signal (i.e., echo) of constant amplitude $A$ changes by a small amount $\Delta \phi$ from pulse to pulse. We wish to find the maximum range of signal phase angles $2 \alpha$ (i.e., $\pm \alpha$ about the median value) beyond which the integrated signal-to-noise ratio would no longer continue to increase as more samples are added. Clearly, $\alpha_{\max }<\pi / 2$, because the signal components of samples obtained when the (relative) phase is near $\pi / 2$ and $-\pi /$ 2 would contribute very little to the summation, while the noise components would continue to add incoherently.

For each sample, the signal component contributes $\left(A \cos \phi_{i}\right)$ to the summation, the relative phase $\phi_{i}$ being measured with respect to the median of the range $2 \alpha$. The orthogonal components $\left(A \sin \phi_{i}\right)$ for corresponding positive and negative values of $\phi_{i}$ sum to zero. Over the range $2 \alpha$ of phase angles there would be $M=2 \alpha$ / $\Delta \phi$ samples, so the signal components add coherently to give an integrated amplitude of

$$
A_{\text {sig }}=\sum_{i=1}^{M} A \cos \phi_{i} .
$$

The power of the integrated signal is therefore proportional to

$$
S=\frac{\grave{A}^{2}}{2}\left[\sum_{i=1}^{M} \cos \phi_{i}\right]^{2} .
$$

At the same time, the $M$ noise components add incoherently to give a noise power proportional to

$$
N=2 \frac{\alpha}{\Delta \phi} e_{n}^{2}
$$

where $e_{n}$ is the root-mean-square noise voltage. Consequently, the signal-to-noise ratio after integration is

$$
\left(\frac{S}{N}\right)_{I}=\frac{A^{2}}{4 e_{n}^{2}}\left[\sum_{i=1}^{M} \cos \phi_{i}\right]^{2} \frac{\Delta \phi}{\alpha} .
$$

If the signal phase did not change at all from pulse to pulse, both $\Delta \phi$ and $\alpha$ would be zero. However, there would still be $M$ samples, each with $\cos \phi_{i}=1$, so the integrated signal-to-noise ratio would be $M$ times that without integration.

To find the maximum value $\alpha_{\max }$ beyond which the integrated signal-to-noise ratio no longer continues to increase, first multiply both the numerator and denominator of (A4) by $\Delta \phi$ :

$$
\left(\frac{S}{N}\right)_{I}=\frac{A^{2}}{4 e_{n}^{2} \alpha \Delta \phi}\left[\sum_{i=1}^{M} \cos \phi_{i} \Delta \phi\right]^{2} .
$$

In the limit of small $\Delta \phi$, the summation can be replaced by an integral with limits $\phi_{i}=-\alpha$ and $\phi_{i}=\alpha$; its value is readily found to be $(2 \sin \alpha)$. Hence

$$
\left(\frac{S}{N}\right)_{I}=\frac{A^{2}}{e_{n}^{2} \Delta \phi} \frac{\sin ^{2} \alpha}{\alpha}
$$

Differentiating (A6) with respect to $\alpha$ and setting the result equal to zero to determine the condition for maximum signal-to-noise ratio, we find

$$
2 \alpha_{\max }=\tan \alpha_{\max } \text {. }
$$

This transcendental equation has the solution

$$
\alpha_{\max }=1.1656 \text { radian }=66.8^{\circ} .
$$

This value can be easily remembered as approximately the same as the latitude of the Arctic Circle.

To express this result in terms of available integration time, note that an echo phase change of $2 \alpha_{\max }=2.331$ radians would be produced by a target radial movement of $0.1855 \lambda$. Therefore the available time for quasi-coherent integration is just

$$
t_{q}=0.1855 \lambda /\left|\bar{v}_{r}\right| \text {. }
$$

The signal-to-noise ratio before integration is just $\left(A^{2} / 2 e_{n}^{2}\right)$. Dividing the ratio after integration, as given by (A6), by this initial value yields the improvement due to the integration. Using $2 \alpha / \Delta \phi=M$, it can be expressed as

$$
F_{I}=\frac{(S / N)_{I}}{\left(A^{2} / 2 e_{n}^{2}\right)}=\frac{\sin ^{2} \alpha}{\alpha^{2}} M
$$

For small $\alpha$, the value of this improvement factor is just $M$, the number of samples integrated, which cor- 
responds to the case of coherent integration. At the maximum condition $\left(\alpha=\alpha_{\max }\right), F_{I}=0.622 \mathrm{M}$, so that quasi-coherent integration is about $62 \%$ as effective as true coherent integration; in other words, it is only 2 $\mathrm{dB}$ less effective.

In the wind profilers described by Strauch et al. (1984), the integration time sometimes is longer than the value given by (A9). It can be as long as $0.25 \lambda / v_{\max }$ when $\bar{v}_{r}=v_{\max }$, the maximum unambiguous Doppler velocity (or can extend up to $\alpha=\pi / 2$ in terms of signal phase). There would seem to be two justifications for this:

(i) For radial speeds less than $(0.1855 / 0.25)=0.74$ times $v_{\max }$, all the integration is beneficial.

(ii) Even when $\bar{v}_{r}=v_{\max }$, the loss in signal-to-noise ratio resulting from extending the integration to $\alpha=\pi /$ 2 , as calculated from (A6), is less than $0.6 \mathrm{~dB}$. For this case, however, (A10) shows that the quasi-coherent in- tegration is only about $40 \%$ as effective as (i.e., $4 \mathrm{~dB}$ less effective than) true coherent integration. A 3-dB improvement could be obtained by reducing the integration time by half (i.e., stopping at $\alpha=\pi / 4$ ) and doubling the size of the sample for the subsequent Fourier transform computation.

\section{REFERENCES}

Gossard, E. E., and R. G. Strauch, 1983: Radar Observations of Clear Air and Clouds. Elsevier, 280 pp.

Nathanson, F. E., 1969: Radar Design Principles. McGraw-Hill, 626 pp.

Smith, P. L., 1986: On the sensitivity of weather radars. J. Atmos. Oceanic Technol., 3, 704-713.

Strauch, R. G., D. A. Merritt, K. P. Moran, K. B. Earnshaw and D. van de Kamp, 1984: The Colorado wind-profiling network. $J$. Atmos. Oceanic Technol., 1, 37-49.

Walker, G. B., P. S. Ray, D. Zrnić and R. Doviak, 1980: Time, angle and range averaging of radar echoes from distributed targets. $J$. Appl. Meteor., 19, 315-323. 